\title{
LiCaNet: Further Enhancement of Joint Perception and Motion Prediction based on Multi-Modal Fusion
}

This paper was downloaded from TechRxiv (https://www.techrxiv.org).

\section{LICENSE}

CC BY 4.0

SUBMISSION DATE / POSTED DATE

01-09-2021 / 08-09-2021

\section{CITATION}

Khalil, Yasser; Mouftah, Hussein T. (2021): LiCaNet: Further Enhancement of Joint Perception and Motion Prediction based on Multi-Modal Fusion. TechRxiv. Preprint. https://doi.org/10.36227/techrxiv.16553580.v1

$\mathrm{DOI}$

10.36227/techrxiv.16553580.v1 


\title{
LiCaNet: Further Enhancement of Joint Perception and Motion Prediction based on Multi-Modal Fusion
}

\author{
Yasser H. Khalil (Member, IEEE), Hussein T. Moutah (Life Fellow, IEEE)
}

\begin{abstract}
(The safety and reliability of autonomous driving pivots on the accuracy of perception and motion prediction pipelines, which in turn reckons primarily on the sensors deployed onboard. Slight confusion in perception and motion prediction can result in catastrophic consequences due to misinterpretation in later pipelines. Therefore, researchers have recently devoted considerable effort towards developing accurate perception and motion prediction models. To that end, we propose LIDAR Camera network (LiCaNet) that leverages multi-modal fusion to further enhance the joint perception and motion prediction performance accomplished in our earlier work. LiCaNet expands on our previous fusion network by adding a camera image to the fusion of RV image with historical BEV data sourced from a LIDAR sensor. We present a comprehensive evaluation to validate the outstanding performance of LiCaNet compared to the state-of-the-art. Experiments reveal that utilizing a camera sensor results in a substantial perception gain over our previous fusion network and a steep reduction in displacement errors. Moreover, the majority of the achieved improvement falls within camera range, with the highest registered for small and distant objects, confirming the significance of incorporating a camera sensor into a fusion network.)
\end{abstract}

Index Terms-Autonomous Driving, Deep Learning, Motion Prediction, Multi-Modal Fusion, Perception, Sensor Fusion.

\section{INTRODUCTION}

The field of autonomous driving had secured incremental progress over the past few years, especially around 2014, when deep learning blossomed. At that time, researchers started regaining hopes that the impediments in autonomous vehicles can be resolved with the help of innovative deep learning. Typically, an autonomous vehicle consists of several pipelines ranging from perception, motion prediction, planning to control [1]. The two pivotal pipelines in autonomous vehicles are perception and motion prediction, as they allow the vehicle to perceive the environment and predict the dynamics in its surroundings. Moreover, all subsequent pipelines rely on the accuracy and robustness of both perception and motion prediction. Without these pipelines, an autonomous vehicle cannot operate safely and reliably. Moreover, fundamental to perception and motion prediction frameworks are the sensors that sense the environment, making them the cornerstone of autonomous vehicles.

In general, an autonomous vehicle is equipped with a suite of different sensors (e.g. LIDAR, camera, ultrasonic, and RADAR) [2]. Although each sensor has advantages and disadvantages, deploying various sensors provides complementary information by reaping the benefits of all deployed sensors while mitigating the inherent challenges of individual sensors. For example, a LIDAR sensor captures accurate depth and physical information of the surrounding environment, and its performance does not get affected under low illumination conditions. A drawback of LIDARs is the enormous number of points they generate, leading to computation and memory bottlenecks. Furthermore, the existence of dust particles in the atmosphere weakens their accuracy. Second, camera sensors are cheap, acquire color information offering rich semantic images, but lack adaptability to poor lighting conditions.

Y. H. Khalil is with the School of Electrical Engineering and Computer Science, University of Ottawa, Ottawa, Canada. (e-mail: ykhal038@uottawa.ca

H. T. Mouftah is with the School of Electrical Engineering and Computer Science, University of Ottawa, Ottawa, Canada. (e-mail: mouftah@uottawa.ca).
Cameras are also ineffective at capturing object ranges and physical sizes. Third, even though ultrasonic sensors can capture depth information, their performance degrades with farther distances. Besides, ultrasonic sensors do not collect semantic information. Lastly, RADARs are robust against varying weather and lighting conditions, strong at detecting objects yet can not be used for classification. Many research works reported challenging results when multi-sensor fusion is applied. Integration of LIDAR and camera sensor is presented in [3], [4], [5], [6], and camera and radar fusion is applied in [7], [8].

On top of multi-sensor fusion, multi-view data representation sourced from one sensor can also be taken advantage of in the fusion process. As LIDARs are the most common sensor used in autonomous driving, we discuss different ways available in the literature to represent LIDAR data. Point clouds can be processed in point-based form [9], 3D voxelization [10], [11], bird's-eye view (BEV) [12], [13], [3], [14], and in range view (RV) [12], [15], [16], [3], [17], [18]. Processing 3D LIDAR point cloud in its raw form is straightforward and requires no transformation. A benefit to this is that no information gets lost. However, the main drawback is the inefficient processing of large number of points. Transforming LIDAR data into $3 \mathrm{D}$ voxelization requires the $3 \mathrm{D}$ points to be discretized into predetermined 3D voxel structures. Each LIDAR point is then mapped to its corresponding voxel. This representation results in a sparse representation as it generates plenty of empty voxels. To avoid the limitations of the previous representations, point clouds are projected into 2D images like BEVs and RV representations. Image projections in $2 \mathrm{D}$ representation are compact, inexpensive to generate, and are efficient to process using 2D convolutions. Also, BEV representation simplifies the process of adding historical information and preserves object sizes making learning easier; but, their resolution is affected by quantization error. One last point to note is that RV form maintains occlusion information. Consequently, it is clear that BEV and RV are the most efficient forms to process LIDAR data and combining them 
offers valuable complementary features being sourced from one sensor.

Multi-modal fusion has become a prominent research direction in the field of autonomous driving. Previous studies presented the benefits of exploiting multi-modal fusion for perception and motion prediction in autonomous vehicles [12], [3], [19], [20]. They showed that applying complementary multi-modal features boosts the performance of perception and motion prediction. In light of the above observations and inspired by [3], we propose LiCaNet, which expands on the fusion network of [12] to involve a camera in addition to a LIDAR. By combining precise range and geometric measurements from a LIDAR sensor and rich semantic information from a camera sensor, we yield a complete set of features. These rich features are later used to generate an enhanced version of joint perception and motion prediction model, offering better accuracy. Thus, LiCaNet involves combining historical LIDAR data encoded in BEV form with RV representation, along with a camera image. Our generated multimodal features are then fed into MotionNet backbone [14] to perform accurate joint perception and motion predictions. MotionNet is a real-time model that perceives and predicts motion at a pixel level with state-of-the-art results. Fig. 1 illustrates the architecture of the proposed LiCaNet model.

To the best of our knowledge, no other study investigates multi-modal fusion network to perform pixel-wise joint perception and motion prediction in real-time for autonomous driving. In summary, the main contribution of this work is proposing a novel fusion scheme that generates an overarching set of features using LIDAR data, in BEV and RV forms, and camera images for perceiving and predicting motion efficiently and effectively. The ultimate goal is to have a real-time and accurate joint perception and motion prediction model that feeds a backbone network vivid and knowledgeable image of the surrounding scene. Such complete features enable the backbone network to make predictions with high confidence, allowing the outcomes to be safely used in later pipelines like planning and control. Conducted experiments prove the superiority of LiCaNet over our previous work [12], MotionNet and thus other state-of-the-art models reported in [14]. LiCaNet achieves outstanding accuracy in real-time for perception and motion prediction, especially for small and distant objects.

The remainder of this paper is organized as follows. Section II provides an overview of the related work. Section III explains our proposed fusion scheme that constitutes historical data in BEV form, RV, and camera data. Also, we provide a brief description of the MotionNet backbone network. The analysis of our performed experiments is discussed in Section IV Finally, Section V concludes our paper.

\section{RELATED WORK}

With the advent of deep learning, significant progress has been made towards perception and motion prediction. In this section, we review the existing prominent literature on perception and motion prediction in the field of autonomous vehicles. Research works have established various methodologies for formulating their input features to feed into the learning models, whether the generated features are sourced from single or multiple sensors. As our proposed work involves two sensors, we target works that deployed a LIDAR, camera, and LIDAR and camera combined.

\section{A. Perception and Motion Prediction using LIDAR Sensor}

There exist many models that depend on a single LIDAR sensor, each representing its input features differently. Some of the prior works processed the raw 3D point cloud directly without any transformations [9], [21], [22]; others transformed the point cloud into 3D voxels [10], [11], [23]. Lately, researchers have considered transforming point clouds into 2D images, such as BEV and RV, for their efficiency in processing and effectiveness in improving the performance of point cloud classification and segmentation [12], [13], [3], [15], [16], [17], [18]. Unlike other representations, LIDAR data projected into $2 \mathrm{D}$ images are more compact and are processed efficiently with the assistance of $2 \mathrm{D}$ convolutions. For example, Shi et al. [21] achieved 3D object detections by directly processing the raw 3D point cloud. PointRCNN [22] is a point-based method that generates $3 \mathrm{D}$ proposals for $3 \mathrm{D}$ object detections. VoxelNet [10] is an end-to-end approach that predicts 3D detections using voxel encodings. Additionally, fast point R-CNN [11] and PV-RCNN [23] performed 3D object detection by incorporating voxel-based and point-based for efficient point cloud feature learning. Other works attained their 3D output detections from 2D image representations of the point cloud. RangeDet [24] proposed an RV-based 3D object detector by considering the scale variations of objects and the inconsistency in the RV image coordinates with the 3D Cartesian coordinates used in outputs. RCD [25] is a method that dynamically adjusts the dilation rate for scale-invariant 3D object detection based on RV images. RV-FuseNet [17] is an end-to-end model that uses time-series input features in RV form to achieve 3D output predictions and motion predictions.

Although perceiving the environment by encoding the point cloud in 3D voxels and generating 3D proposals have their benefits. However, the runtime requirement suffers due to the existence of 3D convolutions and the processing of sparse representations. Normally, point-based models are dataintensive, and so in addition to being time-consuming, they face computation and memory bottlenecks; thus cannot be easily scaled. The following works adopt $2 \mathrm{D}$ input images as input features and perform pixel-wise rather than 3D objectlevel predictions. Khalil et al. [12] proposed combining BEV and RV representations to perceive and predict motion in real-time and in an end-to-end fashion. Khalil et al. [13] used reinforcement learning to train an autonomous vehicle in an urban environment with perception and motion prediction guidance. SqueezeSegV3 [16] used RV images to perceive the driving environment through segmentation. SalsaNext [15] a real-time, uncertainty-aware semantic segmentation model that is based on RV representations. AMVNet [18] used late fusion to perform LIDAR semantic segmentation in RV form. An assertion-guided sampling strategy is used to select uncertain points and extract features of sampled points to feed the point head for improved predictions. Lastly, MotionNet [14] is a real-time BEV-based model that jointly perceives and predicts 
motion at pixel-level with more challenging results than stateof-the-art.

\section{B. Perception and Motion Prediction using Camera Sensor}

Perception algorithms utilizing deep learning and depending on a camera sensor fall under the category of image-based detections based on convolutional neural network (CNN) architectures. Typically, such frameworks are divided into two streams: one-stage and two-stage object detections. Onestage detectors, such as YOLO [26] and SSD [27], directly maps input features to class probabilities and bounding box coordinates via a single-stage CNN model. Whereas twostage detectors, e.g. Faster R-CNN [28] and R-FCN [29], firstly extracts region proposals, then they are refined down the pipeline to generate object classification and regression. In general, one-stage detectors are faster than two-stage detectors but are inferior to two-stage detectors in terms of detection accuracy. An excellent performance assessment of one-stage and two-stage detectors in autonomous driving can be found in [30]. Over the past decade, image-based object detections have picked a staggering pace in the field of autonomous driving, [31], [32], [33].

A typical problem with image-based $\mathrm{CNN}$ detectors is that accuracies for large and small objects are unbalanced. Large objects are represented by sufficient features permitting them to be classified correctly with high confidence. In contrast, small objects are usually represented by inadequate features and thus left undetected or classified with low confidence. In the area of autonomous driving, it is essential to detect small objects (e.g. traffic lights, pedestrians, and bicyclists) to maintain safety and reliability. Recently, researchers proposed image-based CNN algorithms with a focus specifically on detecting small objects. FPN [34] is a two-stage multiscale network that achieved high detection accuracy for small objects. The followed technique is the fusion of multiscale features. PNA [35] is an enhanced version of FPN and specializes in detecting small objects. YOLOv4-5D [31] proposed an improvement to the PNA backbone to increase detection accuracy for small objects. Furthermore, several successful attempts exist for segmentation using camera sensors that resulted in challenging outcomes. Semantic and instance segmentation on street-level datasets was proposed by Porzi et al. [36]. Yang et al. [37] proposed an end-to-end unsupervised learning framework to perform depth estimation and camera motion prediction. Results showed that the utilization of stereo image sequences surpasses scale ambiguity for depth estimation and increases the accuracy of motion prediction for temporal image sequences. It is not common to perform motion prediction using only a camera sensor; however, only lately have camera images been used combined with other sensors to achieve motion prediction.

\section{Fusion of LIDAR and Camera Sensors}

Presently, the utilization of multi-modal fusion for perception and motion prediction has gained much attention among researchers in autonomous driving. Multi-modal fusion is used to exploit the complementary properties of different sensors and representations. Feng et al. [20] presented a survey on different multi-modal methodologies for object detection and segmentation in autonomous vehicles. An in-depth review on the fusion of point clouds and images can be found in [38]. LaserNet++ [4] is an end-to-end model that uses fused features to generate 3D object detection and 3D semantic segmentation. LaserNet++ reported good performance results, especially for small and distant objects. MV3D [19] proposed an end-to-end model to perform 3D object detections and predict motion based on the fusion of LIDAR, camera and HD-maps. Liang et al. [39] used BEV fusion with camera images to perform 3D object detection using a continuous fusion layer. Moreover, Liang et al. [5] achieved an end-to-end 3D object detection using multi-modal fusion by reasoning about 2D and 3D object detection, ground estimation and depth completion. PointPainting [40] applied sequential fusion for semantic segmentation using the painting technique where the point cloud is augmented with image semantics. Later, the $3 \mathrm{D}$ detections are extracted by applying the painted point cloud to a LIDAR detector. MVX-Net [41] proposed 3D object detector using two methods that fuses a point cloud and a camera image in a point-wise or voxel-wise fashion.

In comparison to the reviewed works above, our proposed LiCaNet multi-modal fusion model takes advantage of BEV and RV representations sourced from LIDAR data, in addition to the rich semantics from a camera image. LiCaNet generates a rich and complete set of features that enable improved accuracy for pixel-wise joint perception and motion prediction while maintaining the real-time requirement of autonomous driving. LiCaNet fusion scheme is an extension of [12] that used multi-view fusion sourced from a LIDAR sensor, and backbone network adopted from MotionNet [14].

\section{Proposed Methodology}

This section details the architecture of LiCaNet that combines features from LIDAR and camera sensors. We begin by explaining the formulation of BEV, RV and camera input images. Second, we define the proposed fusion model to generate complementary features. Last, we discuss the backbone network used for learning the generated features to perform accurate pixel-wise joint perception and motion predictions.

\section{A. LIDAR Input Representation}

A LIDAR operates by scanning its entire field-of-view (FOV) using laser beams. The LIDAR measures the time difference between firing a focused laser beam and detecting its reflection. This collected data is used to compute the distance to objects, which can be further used to compute: the xyz-coordinates of objects, the elevation of the beam, and the LIDAR's location.

\section{1) Bird's-Eye View}

Bird's-eye view (BEV) is formed by projecting 3D LIDAR points into 2D images of dimensions $R_{x} \times R_{y} \times H$ meters and grid cell resolution of $\Delta r_{x} \times \Delta r_{y} \times \Delta h$ meters in the xyz-axis. The 2D grid images represent the top-down view of the point cloud, where $R_{x} R_{y} H$ denotes the region-of-interest in the xyz-direction. We set $R_{x}$ and $R_{y}$ to each cover a range 


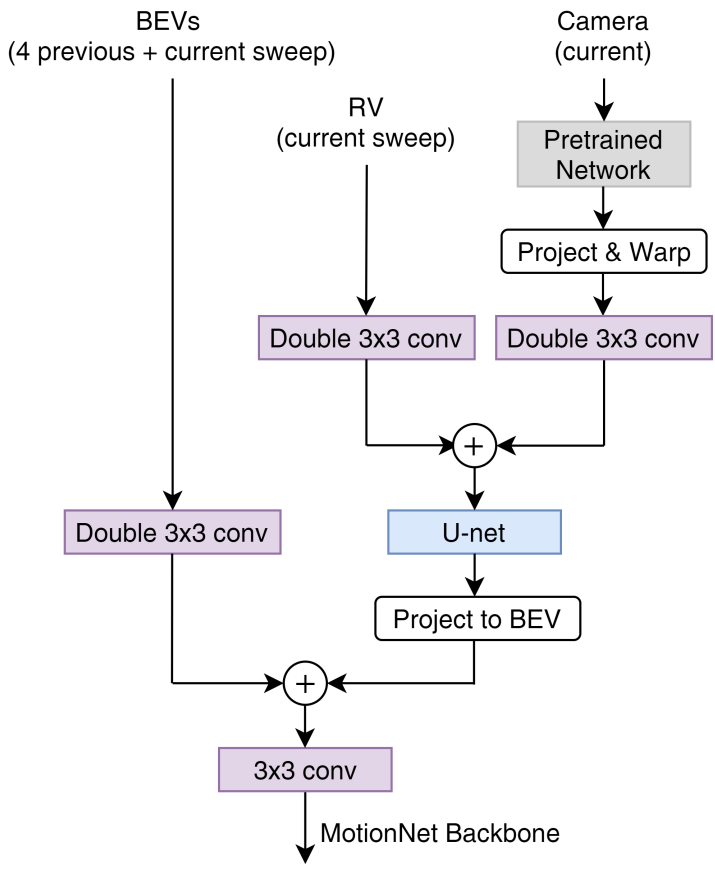

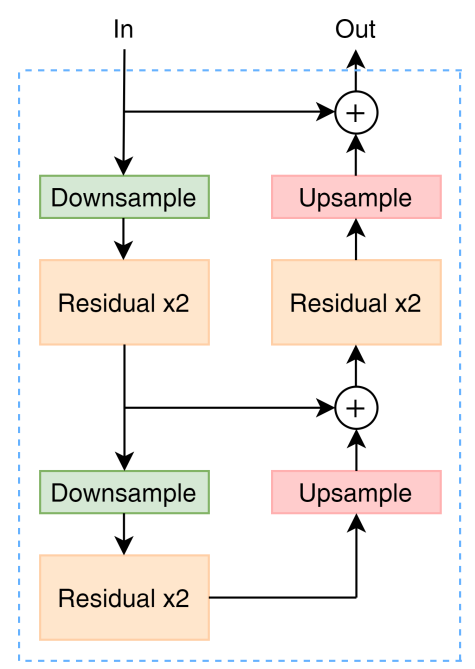

(b) U-Net

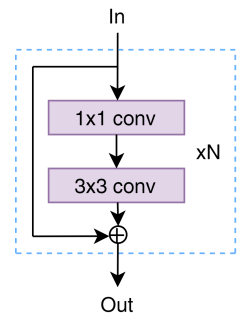

(c) Residual

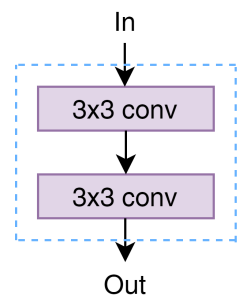

(d) Double $3 \times 3$ conv

(a) LiCaNet Fusion Scheme

Fig. 1: The architecture of our proposed fusion scheme LiCaNet.

of $64 \mathrm{~m}$ in length and width, respectively. The covered range in length is $32 \mathrm{~m}$ long from the front- and back-side of the vehicle $\left(R_{x} \in[-32,32]\right)$. The width range is divided equally between the left- and right-side of the vehicle $\left(R_{y} \in[-32,32]\right)$. Height dimension $H$ covers a total range of 5 meters $(H \in[-3,2])$. We set the resolution of each $2 \mathrm{D}$ grid cell to $\Delta r_{x}=0.25$, $\Delta r_{y}=0.25$ and $\Delta h=0.4$. Discretizing all 3D points into evenly spaced cells results in a BEV image of dimensions $256 \times 256 \times 13$. In other words, the entire height dimension of the point cloud is discretized into 13 image channels $\left(H \Delta h^{-1}\right)$, each with a size of $256 \times 256\left(R_{x} \Delta r_{x}^{-1} \times R_{y} \Delta r_{y}^{-1}\right)$. Upon discretization, a grid cell is considered occupied if at least one point is mapped to it and is marked with a value of 1 ; otherwise, -1 is assigned.

In addition to the current LIDAR sweep, we incorporate a series of historical sweeps to provide the ability to generate motion predictions. We decide to project the historical sweeps into BEV form because of its simplicity in concatenating successive images. So now, we need to convert all historical sweeps into BEV form by discretization. The subsequent step is to synchronize all discretized past sweeps into the present coordinate system of the autonomous vehicle through transformation. The synchronization step is necessary to compensate for the autonomous vehicle motion. We decide on adding 4 past sweeps on top of the current sweep. After the discretization and synchronization steps, all past sweeps in BEV form are added to the current BEV by stacking them on the channel dimension. Thus, the overall BEV input representation becomes $256 \times 256 \times 13 \times 5$.

\section{2) Range View}

Range view (RV) representation is created by projecting each point $p_{i}=(x, y, z)$ in the point cloud to a pixel in the projected RV image. A spherical projection is used to speed up computation. The transformation used for projecting to RV image is defined as follows,

$$
\left(\begin{array}{l}
u_{l} \\
v_{l}
\end{array}\right)=\left(\begin{array}{c}
\frac{1}{2}\left[1-\arctan (y, x) \pi^{-1}\right] w \\
{\left[1-\left(\arcsin \left(z r^{-1}\right)+f_{u p}\right) f^{-1}\right] h}
\end{array}\right),
$$

where $\left(u_{l}, v_{l}\right)$ represents the angular coordinates denoting the pixels in the RV image. $w_{l}$ and $h_{l}$ indicates the width and height of the RV image, respectively. The vertical FOV of the LIDAR sensor is defined as $f=f_{\text {up }}+f_{\text {down }}$, and $r=\sqrt{x^{2}+y^{2}+z^{2}}$ is the range of point $p_{i}$.

We select the projected RV image dimensions to be $1024 \times$ $32 \times 4$. The height value usually reflects the number of LIDAR beams. The LIDAR sensor used in nuScenes dataset [42] is Velodyne HDL32E and has 32 beams. In contrast, the width and channel values are determined by the designer. For each projected point in $(u, v)$, we capture its range $r$, height, intensity, and a binary value indicating if the pixel is occupied by at least one point. Unlike our input BEV representation, our RV form only constitutes the current LIDAR sweep with no historical information. Concatenating past sweeps in RV form requires additional processing than just stacking them on the third dimension.

\section{B. Camera Input Representation}

A camera sensor captures information with color encodings, offering semantically rich images. A front camera is used in this paper that provides RGB images with dimensions $1600 \times$ $900 \times 3$. 


\section{LiCaNet Architecture}

As aforementioned, the significance of fusing multi-modal features is to extract complementary information that contributes to producing improved perception and motion prediction. LIDAR and camera sensors are utilized in LiCaNet to engender a rich set of complementary features. A LIDAR is adopted primarily due to its capability of capturing accurate depth information. Moreover, a front camera is employed for its dense semantic features. To avoid the sparsity of the LIDAR data and the inefficient processing of the vast number of points, LIDAR data is processed in its BEV and RV representations. We select BEV representation to represent LIDAR data because they are handled readily and efficiently by $2 \mathrm{D}$ convolutions. In addition, $\mathrm{BEV}$ representations preserve physical object sizes offering vital prior information to the learning model. Further, a sequence of historical data encoded in BEV form can be easily concatenated. Along with $\mathrm{BEV}$ representations, we use RV images to represent LIDAR data because they are generated from a single viewpoint, making them the most informative means of portraying a point cloud. Another advantage of RV images is that they preserve occlusion information. Consequently, concatenating information from $\mathrm{BEV}, \mathrm{RV}$, and camera representations originates complementary features by leveraging the benefits of all representations.

The architecture of LiCaNet is depicted in Fig. 1 The proposed fusion scheme consists of three main modules. The main module is the LIDAR data in BEV form, the second is the RV module, and the last is the camera module. Starting with the BEV module, once all sweeps are transformed into $\mathrm{BEV}$ representation through discretization and synchronization, the aggregated BEVs are sent down a two-layer $3 \times 3$ convolution layers, named Double $3 \times 3$ conv. At the same time, the RV image and camera features representing only the current timestamp are also passed down a Double $3 \times 3$ conv independently. However, before applying the RGB camera image directly to Double $3 \times 3$ conv, the RGB image is first passed to a small pretrained network. The resulting highlevel features are projected and warped into RV representation. Next, the resulting features from the RV and camera modules are concatenated and applied to a U-net [43]. U-Net is an encoder-decoder network with a strong representation ability mainly because of the skip connections that combine shallow features from the encoder path with deep features from the decoding path at their respective stages. The subsequent step is to concatenate the projected features in BEV representation (from RV and camera modules) with the features from the $\mathrm{BEV}$ module. The last step in the fusion process is to feed the resulting multi-modal features into a single $3 \times 3$ convolution layer. This proposed LiCaNet fusion process generates rich complementary features that enable us to achieve enhanced performance. Finally, the generated multi-modal features are applied to the backbone network to perform accurate pixelwise joint perception and motion prediction. Input features of the LiCaNet are demonstrated in Fig. 2

Directly feeding the fusion network with raw RGB features causes the learning network to discard most features as they

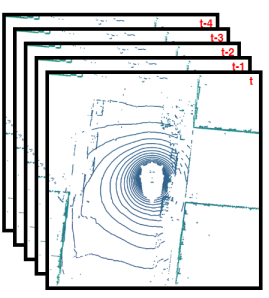

(a) BEV

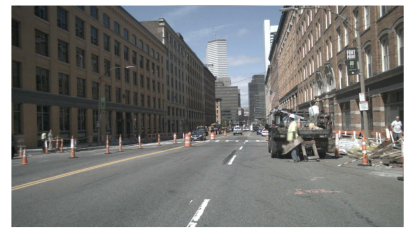

(b) Camera

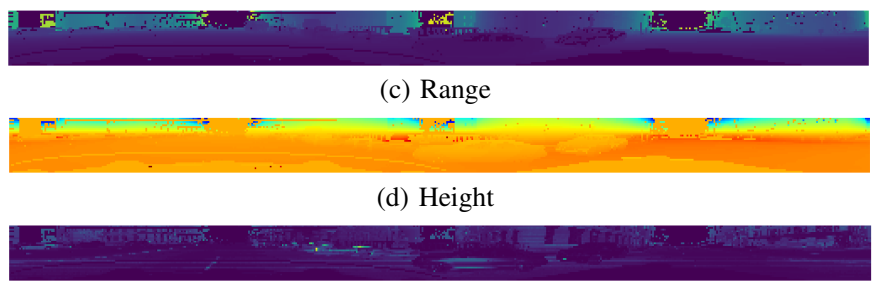

(e) Intensity

Fig. 2: Input features of LiCaNet.

do not comprise valuable high-level information. Thus, the camera image is first passed to a pretrained network to extract high-level features, which are later fed into the fusion network. Now, to use the extracted high-level camera features in our fusion process, we need a mapping from the LIDAR points to the camera image. This mapping permits the retrieval of the high-level camera features corresponding to LIDAR points residing in the camera's FOV. In summary, once we have a mapping from LIDAR points to camera features, we can warp and project features from the camera image into the RV image. The mapping of each LIDAR point $p_{i}$ onto a camera image is achieved by the transformation $T_{c \leftarrow l}$ defined in (2).

$$
T_{c \leftarrow l}=K\left(T_{c \leftarrow v_{t_{c}}} * T_{v_{t_{c}} \leftarrow v_{t_{l}}} * T_{v_{t_{l}} \leftarrow l}\right),
$$

where subscripts $c, l$, and $v$ stands for camera, LIDAR, and vehicle, respectively. $K$ is the intrinsic calibration matrix of the camera. In nuScenes dataset, LIDAR and camera sensors have different operational frequencies and so before transforming LIDAR points to the camera's coordinate system they need to be mapped to the vehicle's coordinate system to compensate for the time-shift between the two sensors. $T_{v_{t_{l}}} \leftarrow l$ transforms LIDAR points to the vehicle's frame at LIDAR capture time $t_{l}$, $T_{v_{t_{c}} \leftarrow v_{t_{l}}}$ transforms the points from vehicle's frame at LIDAR capture time $t_{l}$ to camera capture time $t_{c}$. Last, $T_{c \leftarrow v_{t_{c}}}$ transforms the points from vehicle's frame at $t_{c}$ to the camera's coordinate system. The left image of Fig. 3 shows an example of LIDAR points projected onto a front RGB camera image. The actual mapping is defined in (3).

$$
\left[\begin{array}{lll}
u_{c} & v_{c} & 1
\end{array}\right]^{T}=T_{c \leftarrow l}(p),
$$

where $\left(u_{c}, v_{c}\right)$ are the mapped points from the LIDAR's coordinate system onto the camera.

Eventually, we need to fuse the extracted high-level camera features with RV features. Thus, using the mapping computed in (3) between the LIDAR points and the camera features, we can now project the camera features into RV representation. Up to this point, we assumed that the features extracted from the pretrained network have the same dimensions as the 


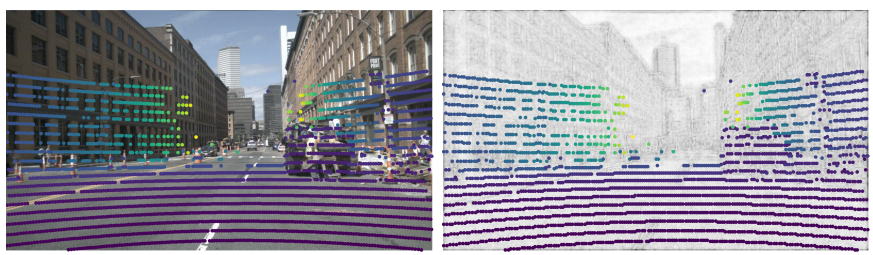

Fig. 3: Examples of LIDAR points projected on camera image. The left image represents the LIDAR points projected on the raw camera image where the one on the right represents the LIDAR points projected on the resulting image of the lightweight pretrained network (in grey scale).

original camera image. Unfortunately, this is not always the case, so to resolve this issue we need to update the mapping between LIDAR points and camera image pixels with a scale factor as follows,

$$
\begin{aligned}
& u_{c_{\text {scaled }}}=u_{c} * s_{h}^{-1} \\
& v_{c_{\text {scaled }}}=v_{c} * s_{w}^{-1}
\end{aligned} \quad \text { and } \quad \begin{aligned}
& s_{h}=\left(h_{c} / h_{h l c f}\right), \\
& s_{w}=\left(w_{c} / w_{\text {hlcf }}\right),
\end{aligned}
$$

where $\left(u_{c_{\text {scaled }}}, v_{c_{\text {scaled }}}\right)$ are the scaled mapped points on the camera image. $\left(h_{c}, w_{c}\right)$ represents the height and width of the original camera image, while $\left(h_{h l c f}, w_{h l c f}\right)$ denotes the resolution of the high-level camera features resulting from the pretrained network. The small pretrained network used to extract high-level features from camera images is described in subsection IV-B.

The projection of features from one form to another is accomplished using the same algorithm followed in [12], which is a painting technique [40]. Briefly, the algorithm takes the mean of all features from the source representation that have correspondence to a LIDAR point $p_{i}$ and projects them to the cell position in the target representation where $p_{i}$ is linked. If no features are projected into a cell of the target representation, then the cell value remains -1 . The algorithm repeats the same process for all points in the point cloud. Fig. 4 demonstrates an illustrative example of the adopted projection algorithm. Primarily, the mapping for each LIDAR point $p_{i}$ is computed in both the source and target form (denoted by blue and orange arrows). Next, all features in the source form where $p_{i}$ is linked to (denoted by $s 1_{p_{i}}, s 2_{p_{i}}$, and $s 3_{p_{i}}$ ) is averaged. Ultimately, the averaged features are positioned in the target's cell where $p_{i}$ is linked (orange arrow). Fig. 5 displays a camera image projected in RV form. The number of LIDAR points projected on the source image determines the resolution of the projected RV image.

\section{Backbone Network}

The backbone network used in this work is called spatiotemporal pyramid network (STPN) from MotionNet [14]. MotionNet is a novel state-of-the-art model that performs pixelwise joint perception and motion prediction competitively and in real-time. MotionNet achieved excellent results when compared with state-of-the-art networks in both perception and motion prediction. Moreover, due to the lightweight of its backbone network STPN, MotionNet is considered one

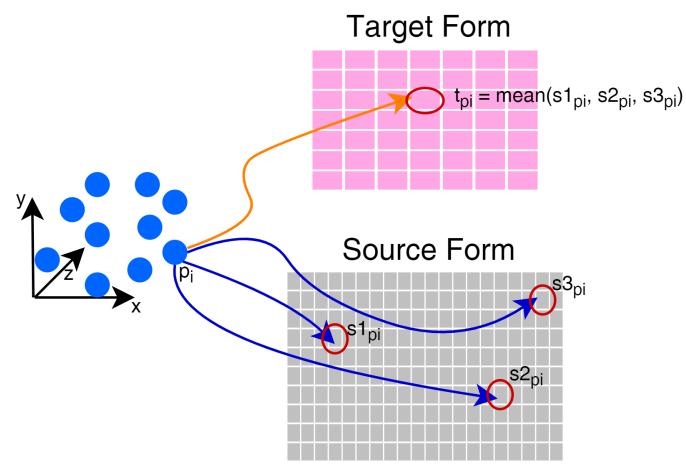

Fig. 4: Illustration of the projection algorithm.

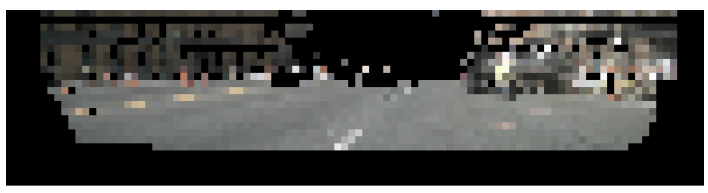

Fig. 5: Example of a front camera image projected into RV representation.

the fastest models for performing joint perception and motion predictions. The reason behind the speed efficiency of MotionNet, is the lightweight backbone STPN which lacks 3D convolutions and uses 2D convolutions instead. The main element of STPN is the spatio-temporal convolution (STC) that constitutes two 2D convolutions followed by one pseudo1D convolution. STPN builds a pyramid with STC blocks to extract features at different stages, leveraging multi-scale spatial and temporal feature learning. Global temporal pooling is used to assist in fusing multi-stage temporal features while going up the pyramid. The design of STPN promotes the extraction of local and global spatio-temporal information. Fig. 6 presents the architecture of STPN.

MotionNet consists of 3 output heads: 1) Cell classification - for perceiving the category of pixels. 2) Motion prediction - for predicting pixels motion. 3) State estimation - for predicting whether the pixels are static or dynamic. MotionNet classifies pixels from 5 different category groups: background, vehicles, pedestrians, bicyclists, and others. The others category is assigned to detected objects that are not categorized in any of the remaining four groups. MotionNet loss function is broken down into six components (5). Three of which are dedicated to globally regularizing network training, and these components are related to the three output heads (cell classification $\mathcal{L}_{\text {class }}$, motion prediction $\mathcal{L}_{\text {motion }}$, and state estimation $\mathcal{L}_{\text {state }}$ ). Spatial consistency loss $\mathcal{L}_{s}$, foreground $\mathcal{L}_{f t}$ and background $\mathcal{L}_{b t}$ temporal consistency loss are the other three components and are dedicated for local regularization.

$$
\mathcal{L}=\mathcal{L}_{\text {class }}+\mathcal{L}_{\text {motion }}+\mathcal{L}_{\text {state }}+\alpha \mathcal{L}_{s}+\beta \mathcal{L}_{f t}+\gamma \mathcal{L}_{\text {bt }},
$$

where $\alpha, \beta$, and $\gamma$ are balancing factors. $\mathcal{L}_{\text {class }}$ and $\mathcal{L}_{\text {state }}$ use weighted cross-entropy loss, while $\mathcal{L}_{\text {motion }}$ uses weighted smooth $L_{1}$ loss. Different weights are used for each class category to counteract the class imbalance issue. $\mathcal{L}_{s}$ uses smooth $L_{1}$ loss to constrain predicted motion between adjacent 


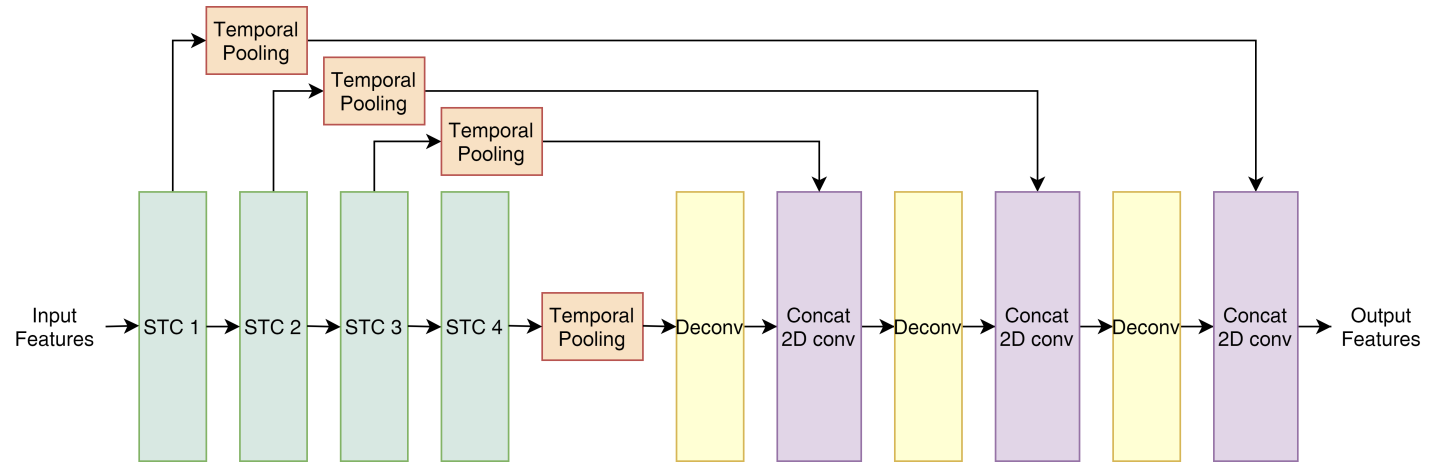

Fig. 6: The architecture of STPN (MotionNet backbone).

pixels of the same object. Similarly, $\mathcal{L}_{f t}$ limits the predicted motion for each object, but temporally rather than spatially. In other words, the motion of objects between consecutive frames should not have abrupt changes. Unlike $\mathcal{L}_{f t}$ that focuses on foreground objects, $\mathcal{L}_{b t}$ concentrates on background cells and tries to minimize the temporal loss of static cells by overlapping them across adjacent frames.

\section{RESUlTS}

This section describes a set of experiments performed to validate the significance of multi-modal fusion for joint perception and motion prediction.

\section{A. Dataset}

The dataset used to conduct our experiments is nuScenes [42]. It consists of 850 scenes, with each having a continuous sequence of sweeps. Our training set consists of 500 scenes (17,065 sequences), while our validation and test set has 100 (1,719 sequences) and 250 scenes (4,309 sequences), respectively. The LIDAR sensor used in the nuScenes dataset is Velodyne HDL32E, consisting of 32 beams, and operates at $20 \mathrm{~Hz}$. The horizontal FOV of the LIDAR is $360^{\circ}$, while the vertical FOV ranges from $-30.67^{\circ}$ to $10.67^{\circ}$. The front camera sensor used in the nuScenes dataset captures images at $12 \mathrm{~Hz}$ with an opening angle of $70^{\circ}$. The resolution of the captured RGB images is $1600 \times 900 \times 3$.

\section{B. Experimental Setup}

We begin our experiments by training MotionNet on our machine to record its potential. Second, we include the experiment conducted in [12] which fuses historical sequence of BEV data with RV representation $(1024 \times 32)$. Subsequently, we repeat the same experiment using a narrowed version of the RV module in the fusion network. Even though LiCaNet minus the camera module is exactly the same as the fusion network in [12]; however, in this paper, we configure the Double $3 \times 3$ conv block of the RV module to encode features in 16 channels. Narrowing down the RV module allows us to fit and train LiCaNet on our machine. This experiment is essential to observe the performance enhancement caused by encompassing the camera module. The next set of experiments analyze the performance of LiCaNet under various lightweight pretrained networks. These experiments are used to monitor whether the performance gain of LiCaNet is consistent across all pretrained networks. All selected lightweight networks dedicated to extracting high-level features from camera images are pretrained on ImageNet. The four pretrained networks investigated are MobileNetv2, VGG16, ResNet50, and ResNeXt50. Only six convolution layers are used from MobileNetv2 and VGG16, and 11 layers from ResNet50 and ResNeXt50.

\section{Training Setup}

The period between consecutive sweeps in forming the sequence of historical BEVs is $0.2 \mathrm{~s}$ apart, and each keyframe characterizing the current sweep is sampled at $2 \mathrm{~Hz}$ for training and $1 \mathrm{~Hz}$ for testing. All experiments are trained with stochastic gradient descent (SGD) and a batch size of 4 . The initial learning rate is set at $1.6 \times 10^{-3}$ and it decays every 10 epochs to end at $0.8 \times 10^{-3}$.

\section{Quantitative Results}

Table I unveils the perception and motion prediction results of our conducted experiments. The evaluation metrics used in this paper are similar to [12] and [14]. For motion prediction evaluation, we divide non-empty pixels into two groups: static and dynamic. Pixels are categorized as static if their predicted motion is 0 ; and dynamic otherwise. Distinguishing static from dynamic pixels is essential because most of the scene has no motion, and combining static and dynamic motion predictions leads to biased displacement errors. Regarding perception evaluation, we report classification accuracy for each category along with the mean classification accuracy (MCA). In addition, we measure the overall pixel accuracy (OA).

It is evident from the collected results in Table [ that all LiCaNet experiments, with different pretrained networks, compare favorably to MotionNet and LiCaNet (LIDAR only) - a narrowed version of the multi-view LIDAR-based fusion network [12]. For fair comparisons, the second experiment in Table I is excluded from our analysis as the depth of its RV module is wider than LiCaNet. Nevertheless, it was included to show that its narrower version has inferior performance in both perception and motion prediction. According to Table I] the use of 6 convolution layers from pretrained VGG16 resulted 
TABLE I: Comparison of perception and motion prediction outcomes between the MotionNet baseline model, LIDAR multiview fusion, and our proposed model LiCaNet. Performance of motion prediction is reported in terms of mean and median $L_{2}$ displacement errors for two groups.

\begin{tabular}{|c|c|c|c|c|c|c|c|c|c|c|c|c|}
\hline Method & \multicolumn{2}{|c|}{ Static } & \multicolumn{2}{|c|}{ Dynamic } & \multicolumn{7}{|c|}{ Classification Accuracy (\%) } & $\begin{array}{r}\text { Time } \\
(\mathrm{ms})\end{array}$ \\
\hline MotionNet [14] & 0.0236 & 0 & 0.4104 & 0.1369 & 97.5 & 91.2 & 74.9 & 22.1 & 65.9 & 70.3 & 96.3 & 20.5 \\
\hline LiCaNet (LIDAR only) & 0.0230 & 0 & 0.4081 & 0.1379 & 97.6 & 92.0 & 80.6 & 22.6 & 69.2 & 72.4 & 96.6 & 28.1 \\
\hline LiCaNet (MobileNetv2_6) & 0.0224 & 0 & 0.4023 & 0.1379 & 97.9 & 92.8 & 82.7 & 23.0 & 70.7 & 73.4 & 96.8 & 30.7 \\
\hline LiCaNet (VGG16_6) & 0.0224 & 0 & 0.4045 & 0.1382 & 97.8 & 92.4 & 84.0 & 23.2 & 71.9 & 73.9 & 96.9 & 31.0 \\
\hline LiCaNet (ResNeXt50_11) & 0.0220 & 0 & 0.4049 & 0.1369 & 98.0 & 92.7 & 81.5 & 23.6 & 70.6 & 73.3 & 96.9 & 33.2 \\
\hline
\end{tabular}

in the best perception, while the use of 11 convolution layers from ResNeXt50 performed best in motion prediction. Even though VGG16 did not attain the lowest displacement errors, however, VGG16 still achieved competitive motion predictions compared to the other pretrained networks. Similarly, the dynamic mean error is not optimal for ResNeXt50 but is still within the competitive range of the other experiments. Now, comparing perception accuracy of LiCaNet (VGG16_6) and LiCaNet (LIDAR only) experiments, we see that the addition of the camera module achieved a substantial increase of $1.5 \%$ and $0.3 \%$ in MCA and OA, respectively. Also, a total gain of $3.6 \%$ in $\mathrm{MCA}$ and $0.6 \%$ in $\mathrm{OA}$ is registered relative to MotionNet baseline.

In addition, examining the classification accuracy per category, we notice that of all the selected pretrained networks, VGG16_6 is considered the best at detecting small objects. With only 6 convolution layers, VGG16_6 was able to secure the highest detection accuracy for pedestrians and competitive accuracy for bikes. In comparison, ResNeXt50_11 used 11 convolution layers to procure the maximum accuracy for Bikes (23.6\%), which is only $0.4 \%$ more than what VGG16_6 accomplished. Also, the use of ResNeXt50_11 did not perform as well as VGG16_6 in detecting objects of the remaining categories. Overall, VGG16_6 outperformed the others at perception. Investigating further classification accuracies reveal that smaller objects have the most significant percentage of perceptual gain compared to the other object groups. LiCaNet (VGG16_6) resulted in a jump of $0.4 \%$ for vehicles, but a rise of $3.4 \%$ and $0.6 \%$ is procured for pedestrians and bikes, respectively, compared to LiCaNet (LIDAR only) experiment.

The presented results in Table II confirm that LiCaNet experiments (for all selected pretrained networks) achieved a tremendous increase in perception and prominent decrease in motion prediction compared to multi-view LIDAR-based fusion model and by default MotionNet baseline too. Thus, we can confidently say that our proposed LiCaNet fusion scheme assists in achieving an enhanced perception and motion prediction model, with the highest enrichment dedicated to small objects.

Table III further investigates the success of LiCaNet experiments by restricting the perception accuracy to within the camera's FOV. The results are also measured based on the distance from the camera sensor. For each category, perception within the camera's FOV is measured at three distance ranges: short-range (S) defined from $0 \mathrm{~m}-10 \mathrm{~m}$, medium-range (M) from $11 \mathrm{~m}-20 \mathrm{~m}$, and far-range $(\mathrm{F})$ from $21 \mathrm{~m}-30 \mathrm{~m}$. Considering the perception accuracy between LiCaNet, MotionNet and LiCaNet (LIDAR only) experiments, we recognize that the accuracy climbs within the camera's FOV for LiCaNet experiments, with the highest rise assigned to small and distant objects.

To begin with, comparing the gain of vehicles between LiCaNet (VGG16_6) and LiCaNet (LIDAR only), we note that a gain of $0.7 \%$ attained for short-range and $1.4 \%$ for far-range. Similarly for pedestrians, an increase of $1.7 \%$ and $1.9 \%$ in short and far-range, respectively. This shows that our proposed model was able to gather higher accuracy for distant objects. Moreover, smaller objects collected even greater gain in perception within the camera's FOV. For example, in the farrange $1.4 \%$ improvement is registered for vehicles, while $1.9 \%$ for pedestrians and $4.0 \%$ for bikes. This proves the potential of LiCaNet in better detecting small and distant objects. Furthermore, generally with any model, the detection accuracy decreases with a farther distance from the sensor; nonetheless, the drop is reduced with LiCaNet. As an example, for LiCaNet (LIDAR only), the accuracy of vehicles, pedestrians, and bikes dropped by $8.4 \%, 6.5 \%$, and $4.8 \%$ between the short- and farrange distance groups, whereas with LiCaNet (VGG16_6), the drop is merely $7.7 \%, 6.3 \%$, and $0.2 \%$; respectively.

Results in Table II showed that perception accuracy of LiCaNet within camera's FOV is substantially better than multiview LIDAR-based fusion network, especially for small and distant objects. Although up to this point, most of our analysis is limited between LiCaNet (VGG16_6) and LiCaNet (LIDAR only), comparing our proposed LiCaNet with MotionNet leads to even more noticeable gains.

Lastly, inference time is compromised when the fusion network is expanded to include a camera module. Thus, a trade-off should be made between accuracy and inference time. In our case, the proposed LiCaNet model (for all selected pretrained networks) met the real-time requirement and is therefore relevant for autonomous driving applications. According to the results in Table $\mathrm{I}$, incorporating a camera module onto our fusion network resulted in a minimum increase of $2.6 \mathrm{~ms}$ using 6 convolution layers from MobileNetv2. VGG16_6 is heavier than MobileNetv2_6, explaining the reason behind an 
TABLE II: Evaluation of classification accuracy within the camera's FOV based on distance ranges from the camera sensor. The three distance groups are: short-range (S) defines the distance from $0 \mathrm{~m}-10 \mathrm{~m}$, medium-range (M) from $11 \mathrm{~m}-20 \mathrm{~m}$, and far-range (F) from $21 \mathrm{~m}-30 \mathrm{~m}$.

\begin{tabular}{|c|c|c|c|c|c|c|c|c|c|c|c|c|c|c|c|}
\hline \multirow{3}{*}{ Method } & \multicolumn{15}{|c|}{ Classification Accuracy in Camera FOV (\%) } \\
\hline & \multicolumn{3}{|c|}{ Background } & \multicolumn{3}{|c|}{ Vehicle } & \multicolumn{3}{|c|}{ Pedestrians } & \multicolumn{3}{|c|}{ Bike } & \multicolumn{3}{|c|}{ Others } \\
\hline & $\mathbf{S}$ & $\mathbf{M}$ & $\mathbf{F}$ & $\mathbf{S}$ & M & $\mathbf{F}$ & $\mathbf{S}$ & $\mathbf{M}$ & $\mathbf{F}$ & $\mathbf{S}$ & $\mathbf{M}$ & $\mathbf{F}$ & $\mathbf{S}$ & M & $\mathbf{F}$ \\
\hline LIDAR fusion [12] & 98.7 & 97.5 & 95.7 & 96.0 & 93.9 & 87.0 & 89.4 & 81.1 & 83.5 & 30.5 & 21.3 & 31.3 & 84.9 & 76.2 & 63.3 \\
\hline LiCaNet (LIDAR only) & 98.6 & 97.1 & 95.2 & 94.5 & 93.3 & 86.1 & 88.6 & 77.0 & 82.1 & 26.2 & 20.1 & 31.0 & 83.4 & 75.2 & 61.7 \\
\hline LiCaNet (MobileNetv2_6) & 98.8 & 97.5 & 95.6 & 95.2 & 94.6 & 87.7 & 90.0 & 86.6 & 84.1 & 28.6 & 21.9 & 38.2 & 83.3 & 75.6 & 66.4 \\
\hline LiCaNet (ResNet50_11) & 98.8 & 97.5 & 95.7 & 94.3 & 87.8 & 87.6 & 89.3 & 78.5 & 83.4 & 26.9 & 22.3 & 34.0 & 86.5 & 75.7 & 66.0 \\
\hline LiCaNet (ResNeXt50_11) & 98.8 & 97.5 & 96.0 & 94.8 & 94.4 & 87.9 & 89.0 & 77.9 & 82.6 & 21.0 & 22.7 & 34.6 & 87.4 & 75.8 & 65.4 \\
\hline
\end{tabular}

additional increase of $0.3 \mathrm{~ms}$. The use of 11 convolution layers will undoubtedly consume extra time compared to 6 layers. Furthermore, ResNeXt50, by default, has a higher inference time as its architecture is denser than ResNet50, this explains why ResNeXt50_11 required more time than ResNet50_11.

\section{E. Qualitative Results}

Qualitative results for LiCaNet are displayed in Fig. 7 We present five different scenes and facilitate visual comparison between the ground truth, LiCaNet (LIDAR only), and LiCaNet (VGG16_6). Each scene is displayed in a column; the first row displays the ground truth, the second row shows LiCaNet (LIDAR only) predictions, and the last row depicts LiCaNet (VGG16_6) predictions. The ground truth is also included in the second and third row for easier visual comparison. Arrows in the examples represent motion prediction. It is clear that the length and direction of arrows predicted by LiCaNet (VGG16_6) are closer to the ground truth compared to LiCaNet (LIDAR only). This indicates that LiCaNet (VGG16_6) achieved better motion prediction. Similarly for perception, the number of correctly categorized pixels in LiCaNet (VGG16_6) examples surpasses LiCaNet (LIDAR only), meaning better perception capabilities. These examples vividly illustrate that our proposed LiCaNet model has enhanced perceptions and motion predictions than LiCaNet (LIDAR only). Therefore, in addition to quantitative analysis, we qualitatively proved that incorporating camera images in the fusion network enhances performance.

Furthermore, Fig. 8 provides visual comparison on perception and motion prediction confined to the camera's FOV. Examining the position of arrows and the classification of pixels, we observe that the accuracy level increases within the camera's FOV, especially for small and distant objects. Thus, this comparison further validates the positive effect of adding camera features to the fusion network.

\section{CONCLUSiON}

We presented a new method, named LiCaNet, that fuses multi-modal features into a backbone network to perform accurate pixel-wise joint perception and motion prediction for autonomous driving. A LIDAR and a camera are used to extract rich and complementary multi-modal features. The proposed LiCaNet uses BEV and $\mathrm{RV}$ forms to represent the LIDAR data. The predictions are attained in real-time, making LiCaNet suitable for the real-world autonomous driving application. This study validated the results of our fusion scheme and demonstrated that the involvement of camera information resulted in challenging results for joint perception and motion prediction and overcomes both MotionNet baseline and multiview LIDAR-based fusion models. In addition, an evident improvement in accuracy was scheduled in the camera's fieldof-view region, with the highest gain recorded for small and distant objects.

\section{REFERENCES}

[1] C. Urmson, J. Anhalt, D. Bagnell, C. Baker, R. Bittner, M. Clark, J. Dolan, D. Duggins, T. Galatali, C. Geyer et al., "Autonomous driving in urban environments: Boss and the urban challenge," in The DARPA urban challenge. Springer, 2009, pp. 1-59.

[2] J. Leonard, J. How, S. Teller, M. Berger, S. Campbell, G. Fiore, L. Fletcher, E. Frazzoli, A. Huang, S. Karaman et al., "A perceptiondriven autonomous urban vehicle," Journal of Field Robotics, vol. 25, no. 10, pp. 727-774, 2008.

[3] S. Fadadu, S. Pandey, D. Hegde, Y. Shi, F.-C. Chou, N. Djuric, and C. Vallespi-Gonzalez, "Multi-view fusion of sensor data for improved perception and prediction in autonomous driving," arXiv preprint arXiv:2008.11901, 2020.

[4] G. P. Meyer, J. Charland, D. Hegde, A. Laddha, and C. VallespiGonzalez, "Sensor fusion for joint $3 \mathrm{~d}$ object detection and semantic segmentation," in Proc. IEEE Conf. Comput. Vis. Pattern Recognit. (CVPR) Workshops, 2019, pp. 0-0.

[5] M. Liang, B. Yang, Y. Chen, R. Hu, and R. Urtasun, "Multi-task multisensor fusion for $3 \mathrm{~d}$ object detection," in Proc. IEEE Conf. Comput. Vis. Pattern Recognit. (CVPR), 2019, pp. 7345-7353.

[6] H. Rashed, M. Ramzy, V. Vaquero, A. El Sallab, G. Sistu, and S. Yogamani, "Fusemodnet: Real-time camera and lidar based moving object detection for robust low-light autonomous driving," in Proc. of the IEEE/CVF Int. Conf. on Computer Vision Workshops, 2019, pp. 0-0.

[7] Z. Liu, Y. Cai, H. Wang, L. Chen, H. Gao, Y. Jia, and Y. Li, "Robust target recognition and tracking of self-driving cars with radar and camera information fusion under severe weather conditions," IEEE Trans. Intell. Transp. Syst., 2021.

[8] X. Dong, B. Zhuang, Y. Mao, and L. Liu, "Radar camera fusion via representation learning in autonomous driving," in Proc. IEEE Conf. Comput. Vis. Pattern Recognit. (CVPR), 2021, pp. 1672-1681.

[9] W. Wu, Z. Qi, and L. Fuxin, "Pointconv: Deep convolutional networks on 3d point clouds," in Proc. IEEE Conf. Comput. Vis. Pattern Recognit. (CVPR), 2019, pp. 9621-9630.

[10] Y. Zhou and O. Tuzel, "Voxelnet: End-to-end learning for point cloud based 3d object detection," in Proc. IEEE Conf. Comput. Vis. Pattern Recognit. (CVPR), 2018, pp. 4490-4499. 


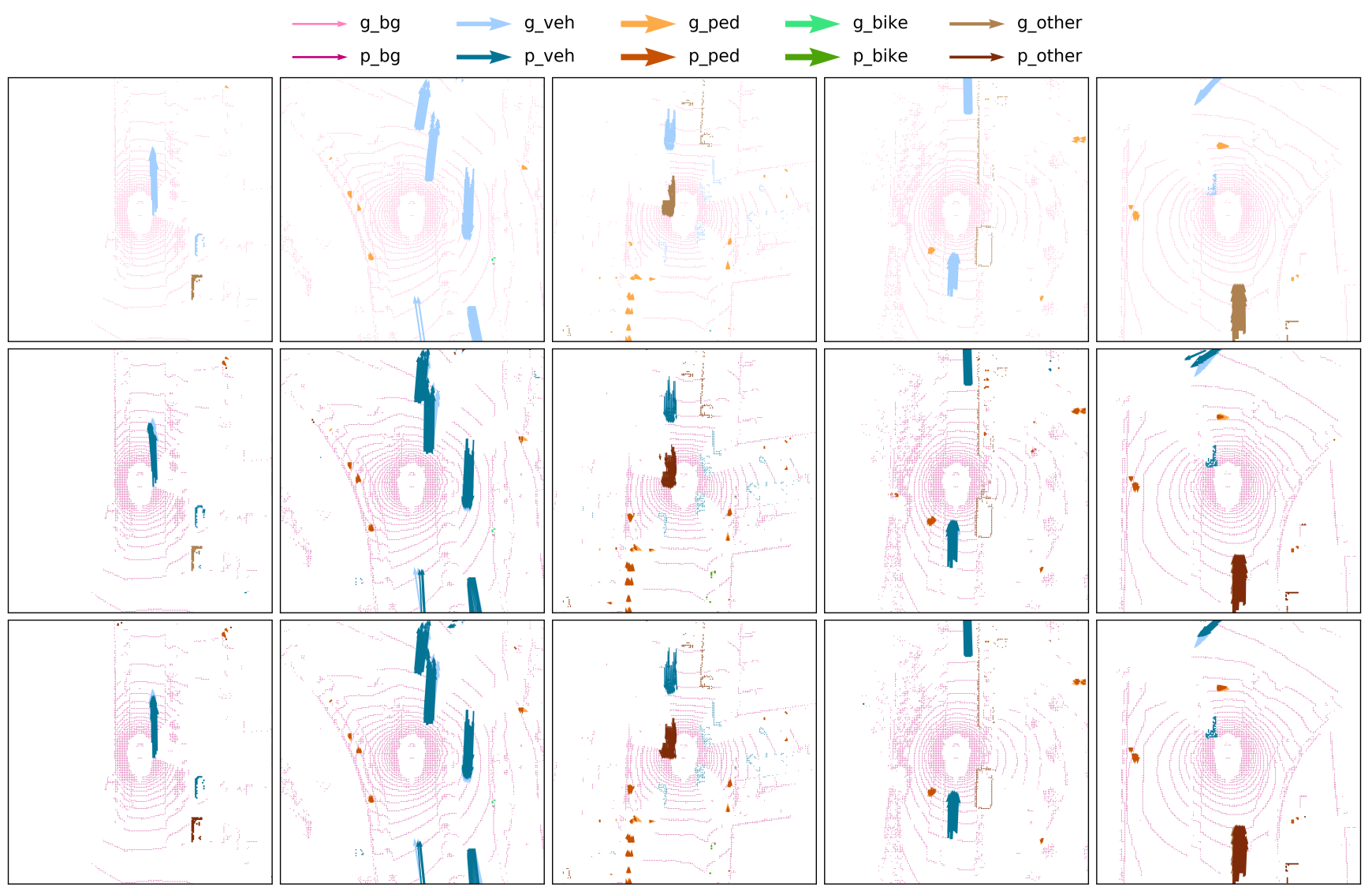

Fig. 7: Qualitative comparison of perception and motion prediction. Top row: ground truth. Middle row: LiCaNet (LIDAR only). Bottom row: LiCaNet (VGG16_6). Ground truth is also present in the second and third row for easier visual comparison. Color codes are presented at the top of the figure. $g_{-}$and $p_{-}$denotes ground truth and prediction colors, respectively.

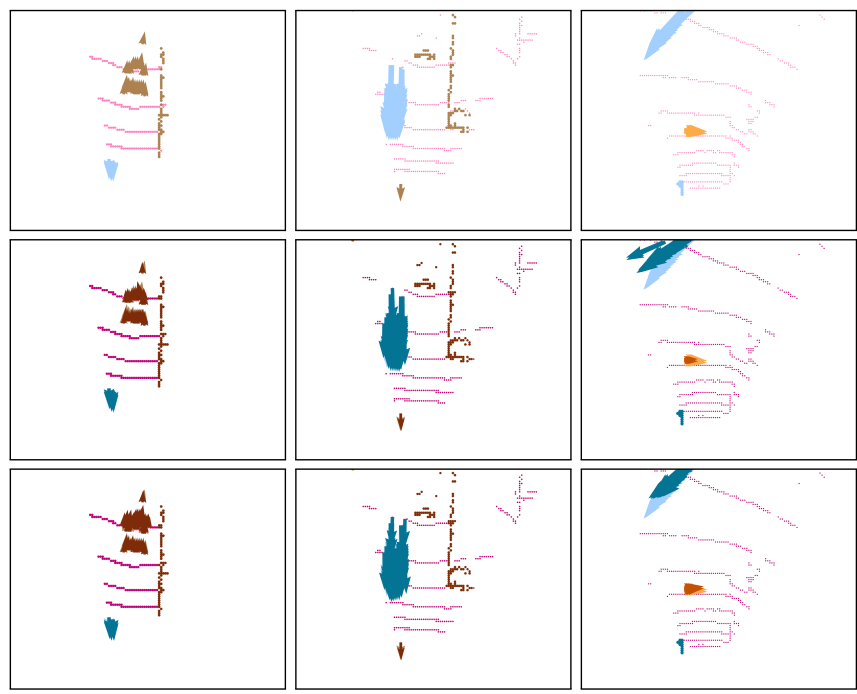

Fig. 8: Examples of perception and motion predictions within camera range. Ground truths are presented in all rows. The second and third rows include the outcomes of LiCaNet (LIDAR only) and LiCaNet (VGG16_6), respectively.
[11] Y. Chen, S. Liu, X. Shen, and J. Jia, "Fast point r-cnn," in Proc. IEEE Int. Conf. on Comput. Vision (ICCV), 2019, pp. 9775-9784.

[12] Y. H. Khalil and H. T. Mouftah, "End-to-end multi-view fusion for enhanced perception and motion prediction," in Proc. 93rd IEEE Veh. Techol. Conf. IEEE, 2021, to be published.

[13] _ "Integration of motion prediction with end-to-end latent RL for Self-Driving vehicles," in Proc. 17th Int. Wireless Comm. Mobile Comp. Conf. (IWCMC) Veh. Comm. Symposium, Harbin, China, 2021.

[14] P. Wu, S. Chen, and D. N. Metaxas, "Motionnet: Joint perception and motion prediction for autonomous driving based on bird's eye view maps," in Proc. IEEE Conf. Comput. Vis. Pattern Recognit. (CVPR), 2020, pp. 11385-11395.

[15] T. Cortinhal, G. Tzelepis, and E. E. Aksoy, "Salsanext: Fast, uncertaintyaware semantic segmentation of lidar point clouds," in Int. Symposium on Visual Computing. Springer, 2020, pp. 207-222.

[16] C. Xu, B. Wu, Z. Wang, W. Zhan, P. Vajda, K. Keutzer, and M. Tomizuka, "Squeezesegv3: Spatially-adaptive convolution for efficient point-cloud segmentation," in European Conf. on Comput. Vision. Springer, 2020, pp. 1-19.

[17] A. Laddha, S. Gautam, G. P. Meyer, C. Vallespi-Gonzalez, and C. K. Wellington, "Rv-fusenet: Range view based fusion of time-series lidar data for joint $3 \mathrm{~d}$ object detection and motion forecasting," arXiv preprint arXiv:2005.10863, 2020.

[18] V. E. Liong, T. N. T. Nguyen, S. Widjaja, D. Sharma, and Z. J. Chong, "Amvnet: Assertion-based multi-view fusion network for lidar semantic segmentation," arXiv preprint arXiv:2012.04934, 2020.

[19] X. Chen, H. Ma, J. Wan, B. Li, and T. Xia, "Multi-view 3d object detection network for autonomous driving," in Proc. IEEE Conf. Comput. Vis. Pattern Recognit. (CVPR), 2017, pp. 1907-1915.

[20] D. Feng, C. Haase-Schuetz, L. Rosenbaum, H. Hertlein, C. Glaeser, F. Timm, W. Wiesbeck, and K. Dietmayer, "Deep multi-modal object detection and semantic segmentation for autonomous driving: Datasets, methods, and challenges," IEEE Trans. Intell. Transp. Syst., 2020.

[21] S. Shi, Z. Wang, J. Shi, X. Wang, and H. Li, "From points to parts: 3d 
object detection from point cloud with part-aware and part-aggregation network," IEEE Trans. Pattern Anal. Mach. Intell., 2020.

[22] S. Shi, X. Wang, and H. Li, "Pointrenn: 3d object proposal generation and detection from point cloud," in Proc. IEEE Conf. Comput. Vis. Pattern Recognit. (CVPR), 2019, pp. 770-779.

[23] S. Shi, C. Guo, L. Jiang, Z. Wang, J. Shi, X. Wang, and H. Li, "Pv-renn: Point-voxel feature set abstraction for 3d object detection," in Proc. IEEE Conf. Comput. Vis. Pattern Recognit. (CVPR), 2020, pp. 10 529-10 538.

[24] L. Fan, X. Xiong, F. Wang, N. Wang, and Z. Zhang, "Rangedet: In defense of range view for lidar-based $3 \mathrm{~d}$ object detection," arXiv preprint arXiv:2103.10039, 2021.

[25] A. Bewley, P. Sun, T. Mensink, D. Anguelov, and C. Sminchisescu, "Range conditioned dilated convolutions for scale invariant $3 \mathrm{~d}$ object detection," arXiv preprint arXiv:2005.09927, 2020.

[26] A. Bochkovskiy, C.-Y. Wang, and H.-Y. M. Liao, "Yolov4: Optimal speed and accuracy of object detection," arXiv preprint arXiv:2004.10934, 2020.

[27] W. Liu, D. Anguelov, D. Erhan, C. Szegedy, S. Reed, C.-Y. Fu, and A. C. Berg, "Ssd: Single shot multibox detector," in European Conf. on Computer Vision. Springer, 2016, pp. 21-37.

[28] S. Ren, K. He, R. Girshick, and J. Sun, "Faster r-cnn: towards real-time object detection with region proposal networks," IEEE Trans. Pattern Anal. Mach. Intell., vol. 39, no. 6, pp. 1137-1149, 2016.

[29] J. Dai, Y. Li, K. He, and J. Sun, "R-fen: Object detection via regionbased fully convolutional networks," arXiv preprint arXiv:1605.06409, 2016.

[30] M. Carranza-García, J. Torres-Mateo, P. Lara-Benítez, and J. GarcíaGutiérrez, "On the performance of one-stage and two-stage object detectors in autonomous vehicles using camera data," Remote Sensing, vol. 13, no. 1, p. 89, 2021.

[31] Y. Cai, T. Luan, H. Gao, H. Wang, L. Chen, Y. Li, M. A. Sotelo, and $\mathrm{Z}$. Li, "Yolov4-5d: An effective and efficient object detector for autonomous driving," IEEE Trans. Instrum. Meas., vol. 70, pp. 1-13, 2021.

[32] X. Hu, X. Xu, Y. Xiao, H. Chen, S. He, J. Qin, and P.-A. Heng, "Sinet: A scale-insensitive convolutional neural network for fast vehicle detection," IEEE Trans. Intell. Transp. Syst., vol. 20, no. 3, pp. 1010-1019, 2018.

[33] D. Guo, L. Zhu, Y. Lu, H. Yu, and S. Wang, "Small object sensitive segmentation of urban street scene with spatial adjacency between object classes," IEEE Trans. Image Process., vol. 28, no. 6, pp. 2643-2653, 2018.

[34] S. Liu, L. Qi, H. Qin, J. Shi, and J. Jia, "Path aggregation network for instance segmentation," in Proc. IEEE Conf. Comput. Vis. Pattern Recognit. (CVPR), 2018, pp. 8759-8768.

[35] C. Ma, Y. Guo, Y. Lei, and W. An, "Binary volumetric convolutional neural networks for 3-d object recognition," IEEE Trans. Instrum. Meas., vol. 68, no. 1, pp. 38-48, 2018.

[36] L. Porzi, S. R. Bulo, A. Colovic, and P. Kontschieder, "Seamless scene segmentation," in Proc. IEEE Conf. Comput. Vis. Pattern Recognit. (CVPR), 2019, pp. 8277-8286.

[37] D. Yang, X. Zhong, D. Gu, X. Peng, and H. Hu, "Unsupervised framework for depth estimation and camera motion prediction from video," Neurocomputing, vol. 385, pp. 169-185, 2020.

[38] Y. Cui, R. Chen, W. Chu, L. Chen, D. Tian, Y. Li, and D. Cao, "Deep learning for image and point cloud fusion in autonomous driving: A review," IEEE Trans. Intell. Transp. Syst., 2021.

[39] M. Liang, B. Yang, S. Wang, and R. Urtasun, "Deep continuous fusion for multi-sensor 3d object detection," in Comput. Vis. - ECCV 2018. Cham: Springer International Publishing, 2018, pp. 663-678.

[40] S. Vora, A. H. Lang, B. Helou, and O. Beijbom, "Pointpainting: Sequential fusion for $3 \mathrm{~d}$ object detection," in Proc. IEEE Conf. Comput. Vis. Pattern Recognit. (CVPR), 2020, pp. 4604-4612.

[41] V. A. Sindagi, Y. Zhou, and O. Tuzel, "Mvx-net: Multimodal voxelnet for 3d object detection," in 2019 Int. Conf. on Robotics and Automation (ICRA). IEEE, 2019, pp. 7276-7282.

[42] H. Caesar, V. Bankiti, A. H. Lang, S. Vora, V. E. Liong, Q. Xu, A. Krishnan, Y. Pan, G. Baldan, and O. Beijbom, "nuscenes: A multimodal dataset for autonomous driving," in Proc. IEEE Conf. Comput. Vis. Pattern Recognit. (CVPR), 2020, pp. 11621-11631.

[43] O. Ronneberger, P. Fischer, and T. Brox, "U-net: Convolutional networks for biomedical image segmentation," in Int. Conf. on Medical Image Computing and Computer-Assisted Intervention. Springer, 2015, pp. 234-241.

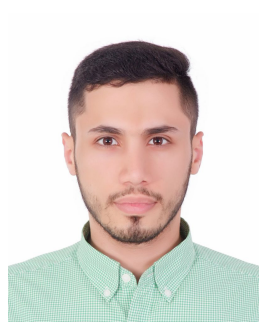

Yasser H. Khalil (Member, IEEE) received the BEng (Hons.) in Computer Engineering from the American University of Kuwait in 2015, and the MSc degree (Hons.) from Kuwait University in 2019. He is currently pursuing the $\mathrm{PhD}$ degree in Electrical and Computer Engineering at the University of Ottawa. He has two years of industrial experience and over four year in academia. He is currently appointed as a research assistant at the University of Ottawa. His current research interests include artificial intelligence, deep learning, reinforcement learning, autonomous driving, sensor fusion, perception and motion prediction.

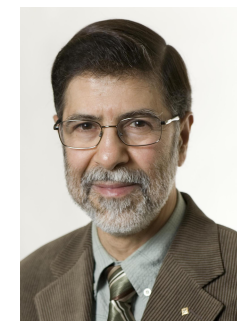

Hussein T. Mouftah (Life Fellow, IEEE) received the BSc in Electrical Engineering and MSc in Computer Science from the University of Alexandria, Egypt, in 1969 and 1972, respectively, and the $\mathrm{PhD}$ in Electrical Engineering from Laval University, Canada, in 1975. He joined the School of Electrical Engineering and Computer Science (was School of Information Technology and Engineering) of the University of Ottawa in 2002 as a Tier 1 Canada Research Chair Professor, where he became a Distinguished University Professor in 2006. He has been with the ECE Dept. at Queen's University (1979-2002), where he was prior to his departure a Full Professor and the Department Associate Head. He has six years of industrial experience mainly at Bell Northern Research of Ottawa (Nortel Networks). He served as Editor-in-Chief of the IEEE Communications Magazine (1995-97) and IEEE ComSoc Director of Magazines (1998-99), Chair of the Awards Committee (2002-03), Director of Education (200607), and Member of the Board of Governors (1997-99 and 2006-07). He has been a Distinguished Speaker of the IEEE Communications Society (20002007). He is the author or coauthor of 13 books, 73 book chapters and more than 1800 technical papers, 17 patents, 6 invention disclosures and 148 industrial reports. He is the joint holder of 25 Best/Outstanding Paper Awards. He has received numerous prestigious awards, such as the C. Gotlieb Medal in Computer Science and Engineering, the 2016 R.A. Fessenden Medal in Telecommunications Engineering of IEEE Canada, the 2015 IEEE Ottawa Section Outstanding Educator Award, the 2014 Engineering Institute of Canada K. Y. Lo Medal, the 2014 Technical Achievement Award of the IEEE Communications Society Technical Committee on Wireless Ad Hoc and Sensor Networks, the 2007 Royal Society of Canada Thomas W. Eadie Medal, the 2007-2008 University of Ottawa Award for Excellence in Research, the 2008 ORION Leadership Award of Merit, the 2006 IEEE Canada McNaughton Gold Medal, the 2006 EIC Julian Smith Medal, the 2004 IEEE ComSoc Edwin Howard Armstrong Achievement Award, the 2004 George S. Glinski Award for Excellence in Research of the University of Ottawa Faculty of Engineering, the 1989 Engineering Medal for Research and Development of the Association of Professional Engineers of Ontario, and the Ontario Distinguished Researcher Award of the Ontario Innovation Trust. Dr. Mouftah is a Fellow of the IEEE (1990), the Canadian Academy of Engineering (2003), the Engineering Institute of Canada (2005) and the Royal Society of Canada RSC Academy of Science (2008). 